\title{
The localization and adsorption of benzene and propylene in ITQ-1 zeolite: grand canonical Monte Carlo simulations
}

\author{
T.J. Hou, L.L. Zhu, Y.Y. Li, X.J. Xu* \\ College of Chemistry and Molecular Engineering, Peking University, Beijing 100871, People's Republic of China
}

Received 27 March 2000; accepted 6 April 2000

\begin{abstract}
Grand canonical Monte Carlo (GCMC) simulations have been performed to investigate the localization and adsorption behavior of benzene and propylene, which are involved in the cumene synthesis process, in purely siliceous MWW zeolite (ITQ-1). From the mass clouds of GCMC simulations, it can be seen that the benzene and propylene molecules show different localization and adsorption behavior in the zeolite cavities. In the 10-MR channels, both benzene and propylene show high localization. In the 12-MR supercages, the propylene molecules cannot only almost fill all the possible positions in one supercage, but also can be steadily located in the short 10-MR conducts interconnecting the 12-MR supercages, where the benzene molecules are adsorbed close to three adsorption sites. By analyzing the location of benzene and propylene in ITQ-1, it can be deduced that the alkylation of benzene and propylene will happen mainly in 12-MR supercages. Moreover, a series of simulations have been performed to predict the adsorption isotherms of benzene and propylene at $315 \mathrm{~K}$ and $0-1.4 \mathrm{kPa}$. The results for benzene generally are in agreement with the trend from experiments on a series of aromatic compounds. The results reveal that at low pressures, the loading of propylene is lower than that of benzene, which seems to be caused by the relatively unfavorable potential interactions between propylene/zeolite and propylene/propylene. (C) 2001 Elsevier Science B.V. All rights reserved.
\end{abstract}

Keywords: ITQ-1; Grand canonical Monte Carlo simulations; Adsorption; Benzene; Propylene

\section{Introduction}

The alkylation of benzene with propylene to produce cumene, a starting material for the production of acetone and phenol [1], is very important in hydrocarbon processes. Traditionally, some mineral acids including $\mathrm{AlCl}_{3}$ and others are employed, which present good catalytic performance [2,3], while raising serious environmental problems such as corrosion and waste disposal. In order to overcome some drawbacks of the traditional mineral acid catalysts, tech-

\footnotetext{
* Corresponding author.

E-mail address: xiaojxu@chemms.chem.pku.edu.cn (X.J. Xu).
}

nologies such as the Mobil-Badger process using $\mathrm{H}-$ ZSM-5 [4], the CDTEC and ENI process using Y and $\beta$ zeolites [5] and the DOW process using Mordenite [6] have made great progress in recent years. The zeolites are considered to be cleaner catalysts; moreover, they can effectively reduce the amount of less desired products such as diisopropylbenzenes, but introduce the formation of $n$-propylbenzene which is not formed in significant amounts when using mineral acid catalysts [7].

It is well known that in the case of zeolites, the pore dimensions and the diffusion of the reactants and products significantly contribute to reactions that occurred in zeolites, since the reactivity and final 
product distributions will depend highly on the zeolite channel structure and the diffusion of the sorbate molecules. For instance, the formation of $n$-propyltoluene has been observed with ZSM-5 but not with Mordenite, which suggests that the transalkylation reaction occurs in the 10-MR channel intersection of ZSM-5, whereas the intersection of 12-MR and 8-MR channels in Mordenite does not provide sufficient space for the bimolecular transalkylation process to occur. However, the yield of transalkylation in zeolite $\mathrm{Y}$ is found to be much lower than that in ZSM-5 under the same reaction conditions, which suggests that the reactivity cannot be determined only by the size of cavities in which the reactions take place, but the diffusion of the chemical compounds involved in the reaction also needs to be taken into account. With respect to energy, the energetic complementarity between the zeolite and the chemical compounds involved in the reaction is simply expressed as shape adsorption or shape selectivity of the zeolite. The diffusion of the adsorbents in the zeolite is really a "docking" process, and they always prefer to follow an energetically favorable pathway.

Several zeolites including H-ZSM-5, USY, $\beta$ and MCM-22 have been tested for the alkylation of benzene with propylene, and it has been found that MCM-22 is very reactive [8]. Zeolite MCM-22 (IZA code MWW) is a novel zeolite discovered recently by scientists at Mobil $[9,10]$. Compared with other common types of zeolites, MCM-22 possesses an interesting and unusual framework structure: two independent pore systems formed by interconnected sinusoidal 10 -MR pores with a 4-5.5 ̊ diameter and an independent 12-MR supercage with 18.2-7.1 linked by $10-\mathrm{MR}$ windows. The unusual framework topology, high thermal stability, large surface area and good adsorption capacity render this zeolite very interesting for catalysis. Several previous studies have looked into the diffusivities of benzene and cumene in the MWW structure [11,12]. It has been found that the diffusion and adsorption behaviors of these molecules are different in the two independent pore systems. The simulations of Perego et al. [11] indicated that the diffusion of cumene was more favorable in the 12-MR supercages than in the 10MR sinusoidal system, although the 10-MR windows interconnecting the 12-MR supercages still presented a diffusion barrier for cumene. The molecular dynamics simulations of Sastre et al. [12] revealed that benzene was not seen to diffuse through two pore systems, and only intracage mobility was seen in the supercage voids where a certain activation energy must be necessary for a benzene molecule migrating between 12-MR supercages through 10MR windows.

Until now, few studies have been performed to investigate the localization and adsorption of benzene and propylene in MCM-22, and the favorable adsorption sites of those molecules in zeolite lattices are not clear. As far as we know, no work has been undertaken yet to investigate the adsorption properties of zeolite and propylene in MWW type zeolites through grand canonical Monte Carlo simulations (GCMC) simulations. In the present work, the GCMC simulation technique was used to predict the adsorption characteristics of benzene and propylene in the ITQ-1 zeolite. We intended to determine the potential adsorption sites of benzene and propylene in ITQ-1 lattice, as well as the properties of the localization of benzene and propylene in the zeolite. In the meantime, we wanted to predict the adsorption isotherms for these two kinds of molecules.

\section{Method}

\subsection{Model representations and potential forcefield}

Considering the high Si/Al ratio of the MCM-22 type zeolite and the difficulty of determining $\mathrm{Al}$ distributions in disordered zeolites by experiments, meanwhile, in order to simplify the simulations, the pure siliceous analogue of MCM-22, ITQ-1, was adopted in this paper. The model of the ITQ-1 was constructed according to the results from Camblor et al. [13]. In the simulations, the silicon and oxygen atoms of the zeolite framework were assumed to be fixed at their crystallographic positions from X-ray diffraction studies. The benzene and propylene molecules were rigid, that is to say, they could only translate and rotate, but were not allowed to deform.

The zeolite and the sorbates were assumed to interact via a pairwise-additive potential between atoms of the guest molecules and atoms of the zeolite. The site-site interactions are models with a 
Table 1

Lennard-Jones parameter for six types of atoms

\begin{tabular}{lll}
\hline Atom type & $D_{0}\left(\mathrm{kcal} \mathrm{mol}^{-1}\right)$ & $R_{0}(\AA)$ \\
\hline O_z & 0.1648 & 3.3000 \\
Si_z & 0.0496 & 4.2000 \\
C_R & 0.0951 & 3.8983 \\
C_3 & 0.0951 & 3.8983 \\
C_2 & 0.0951 & 3.8983 \\
H & 0.0152 & 3.1950 \\
\hline
\end{tabular}

Lennard-Jones plus Coulomb potential,

$V\left(r_{i j}\right)=D_{i j}\left\{\left[\frac{\left(R_{0}\right)_{i j}}{R_{i j}}\right]^{12}-2\left[\frac{\left(R_{0}\right)_{i j}}{R_{i j}}\right]^{6}\right\}+\frac{q_{i} q_{j}}{R_{i j}}$

where $i$ and $j$ indicate atoms of the sorbate and of the zeolite, respectively, and $R_{i j}$ is the distance between them. $D_{i j}$ and $\left(R_{0}\right)_{i j}$ are the Lennard-Jones parameters, and $q_{i}$ and $q_{j}$ are the partial charge of the atoms. Six different atom types were considered in the studied system, including $\mathrm{O} \_\mathrm{z}$ (O atom in zeolite framework), $\mathrm{Si}$ ( $\mathrm{Si}$ atom in zeolite framework), C_R (C atom in benzene ring), C_3 $\left(\mathrm{sp}^{3} \mathrm{C}\right.$ in propylene), $\mathrm{C} \_2$ ( $\mathrm{sp}^{2} \mathrm{C}$ in propylene) and $\mathrm{H}$ ( $\mathrm{H}$ atom in benzene and propylene). The Lennard-Jones parameters for O_z and Si originally derived by Burchart [14], and those for other atom types taken from Mayo [15], are listed in Table 1. Then, the off-diagonal van der Waals parameters for each pair of atoms were calculated based on the geometric mean. The partial charges for $\mathrm{O}_{-} \mathrm{z}$ $(-0.19|e|)$ and $\mathrm{Si}(+0.38|e|)$ were taken from the calculations of Burchart. The above hybrid forcefield has been tested by MSI and distributed as a BurchartDreiding force field [16]. The partial charges for the atoms in benzene and propylene (Fig. 1) were computed using the AM1 method, available in MOPAC 7.0 [17].

\subsection{Grand canonical Monte Carlo simulations}

The GCMC simulation may be the most common technique for predicting the zeolite adsorption phase equilibria from molecular simulations [18-20]. The GCMC simulation technique simulates the equilibrium of a collection of adsorbates in a micropore at constant chemical potential, volume, and temperature or pressure. In the GCMC simulations, the number of particles in the system is not fixed, but the chemical potential of each species is fixed. Sorption translates the chemical potential into the partial pressure (or fugacity) of each component. Equilibrium is achieved when the temperature $\left(T_{\text {frame }}\right)$ and the chemical potential $\left(\mu_{\text {frame}}\right)$ of the gas inside the framework are equal to the temperature $\left(T_{\mathrm{gas}}\right)$ and chemical potential $\left(\mu_{\mathrm{gas}}\right)$ of the free gas outside the framework. For a non-ideal gas, the chemical potential depends upon fugacity $(f)$, which is a function of both temperature and pressure. The bulk pressure can be determined from the chemical potential using a Lennard-Jones equation. So the GCMC simulation technique enables one to study many important characteristics of zeolite systems under certain pressure and temperature.

Eight unit cells of zeolite with a total of 1728 atoms were used to construct the simulation box, and periodic boundary conditions were applied in three dimensions in order to simulate an infinite (macroscopic) system. In order to achieve the real equilibration of the system, the length of the simulations was totally $6 \times 10^{6}$ steps, and every 600 steps, a configuration of the system was recorded. The first three million steps were used for equilibration and not included in

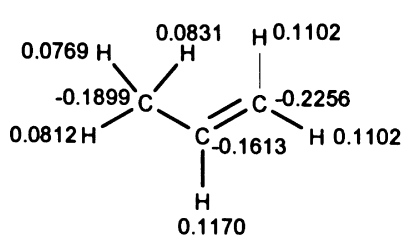

(b)

Fig. 1. Partial charges for: (a) benzene; and (b) propylene used in calculating the zeolite/sorbates and sorbates/sorbates interaction energy, given in units of the electron charges $|e|$. 


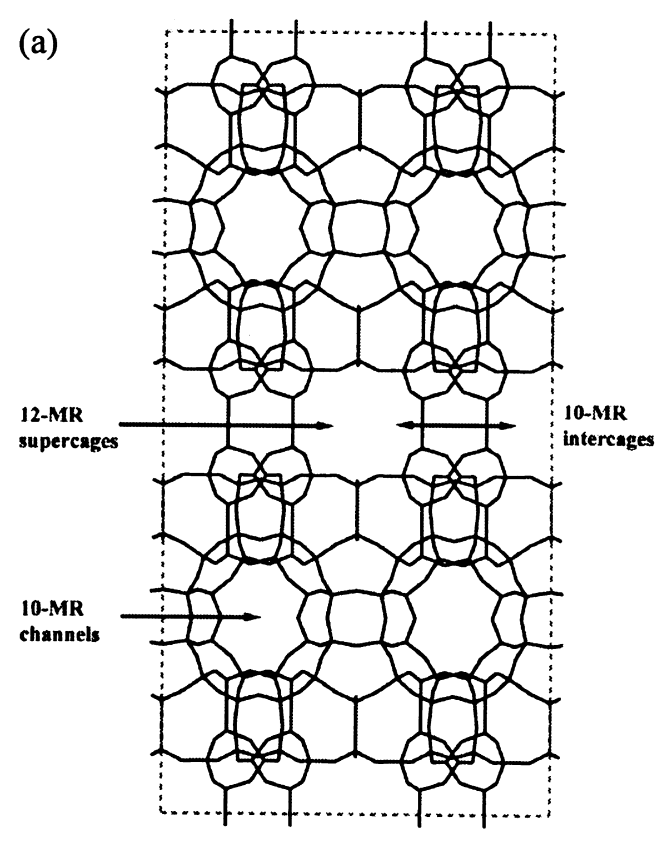

(b)

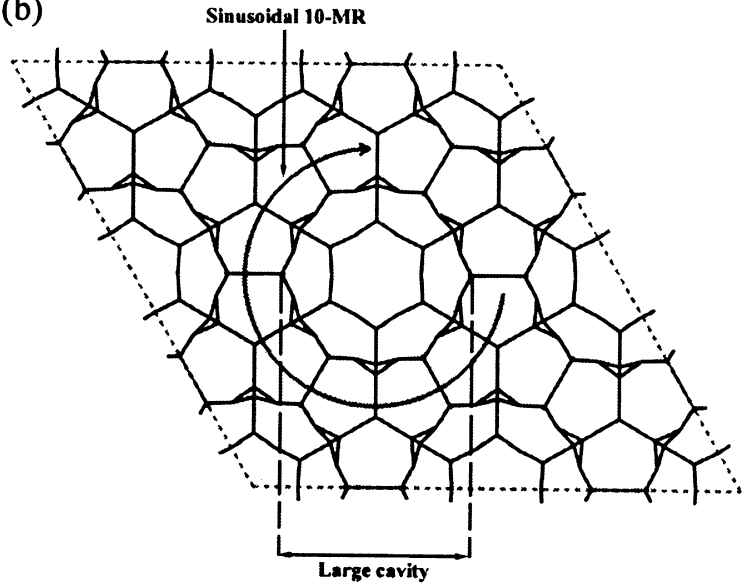

(c)

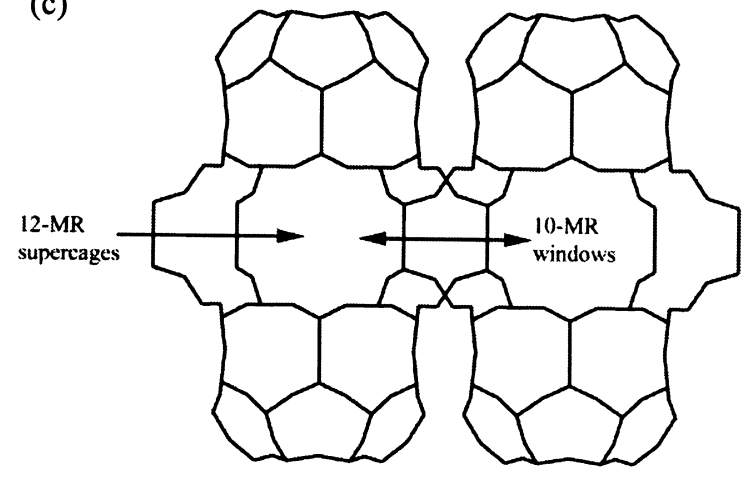

Fig. 2. Skeletal drawings of the framework structure of ITQ-1 (MWW structure type). (a) Schematic view of the independent pore systems along the $y z$ axis. (b) Schematic view of the independent pore systems along the $x y$ axis. (c) Two adjacent 12-MR supercages. The sinusoidal 10-MR channels are all interconnected to each other, and multiple diffusion trajectories can be allowed to every diffusing molecule. The 12-MR are independent with 10-MR channels, which are interconnected through short 10-MR windows. 
the averaging. A cutoff of $10 \AA$ was applied to the Lennard-Jones interactions, and the long-range electrostatic interactions were calculated by using the Ewald summation technique. The Ewald summation to calculate the adsorbate-adsorbent and adsorbateadsorbate is generally time-consuming. We therefore used a grid-interpolation procedure in which the simulation boxes were split into a collection of small cubes. The grid-interpolation method allows us to take into account any degree of accuracy in the description of the adsorbate/zeolite and adsorbate/ adsorbate interaction energy since all the needed grids are calculated separately prior to any simulation runs. First, the GCMC simulations were carried out in the condition of $300 \mathrm{~K}$ and $1 \mathrm{~atm}$. Then, a series of simulations were performed to predict the adsorption isotherms for benzene and propylene at $315 \mathrm{~K}$. All calculations were performed in the CERIUS ${ }^{2}$ molecular simulation package [16] on a SGI Octane 2-CPU workstation.

In this paper, we also computed the potential energy of the diffusing benzene molecules along their trajectory, which allowed estimation of the energy profiles of one benzene molecule while it diffused between two adjacent 12-MR supercages through 10-MR windows in the ITQ-1 zeolite. Consequently, the activation energy of one benzene molecule through 10-MR windows was predicted.

\section{Results and discussion}

A deep insight into the channel systems present in ITQ-1 (Fig. 2) reveals some special features that will greatly affect the location and adsorption of sorbates in the zeolite lattice. First, all 10-MR sinusoidal channels are interconnected with each other and have high tortuosity. The benzene molecules and even the propylene ones may be restricted through sinusoidal channels in ITQ-1. Second, the larger 12-MR cavities have large dimensions with $7.1 \AA \times 18.2 \AA$, so they are expected to host both benzene and propylene whose mobility and location inside the supercages will be very interesting to investigate. Additionally, we are also interested in knowing whether they migrate from one cavity to another nearby through 10-MR openings or tend to remain inside a given cavity. Certainly, all these features will depend on
Table 2

The cell parameters and the GCMC simulation results for four models

\begin{tabular}{llllll}
\hline Model & $a(\AA)$ & $b(\AA)$ & $c(\AA)$ & Loading & $E\left(\mathrm{~kJ} \mathrm{~mol}^{-1}\right)^{\mathrm{a}}$ \\
\hline 1 & 14.2081 & 14.2081 & 24.9452 & 59.71 & -81.9 \\
2 & 14.3502 & 14.3502 & 24.9452 & 58.14 & -81.1 \\
3 & 14.4212 & 14.4212 & 24.9452 & 62.55 & -82.3 \\
4 & 14.2081 & 14.2081 & 25.4410 & 60.11 & -80.7 \\
\hline
\end{tabular}

${ }^{a}$ The average interaction energy between benzene and the zeolite framework.

the conditions of the environment including temperature and pressure.

\subsection{The influence of the minor distortions of framework to simulation results}

In the calculations, the zeolite atoms were fixed at their crystallographic positions. Generally, the framework of zeolite is relatively rigid and possesses good thermal stability. For instance, the introduction of xylene into the cavities of the faujasite $\mathrm{NaY}$ has little effect on the framework structure [21]. In order to examine the influence of the minor change of zeolite lattice on the simulation results, the framework of the ITQ-1 zeolite was manually adjusted to obtain three transformative structures: the first structure was obtained by expanding (by averages) the coordinates of all atoms by $1 \%$ along the $a$ and $b$ directions; the second one was obtained by expanding (by averages) the coordinates by $1.5 \%$ along the $a$ and $b$ directions; and the third one was obtained by expanding (by averages) the coordinates by $2 \%$ along the $c$ direction. Compared with the volume of the crystallographic structure, the volumes of these three adjusted structures are 1, 2.75 and 2\% larger (the cell parameters for these three adjusted models are listed in Table 2). For these three adjusted structures and the crystallographic structure, the simulations of benzene in ITQ-1 were performed by using GCMC simulations at standard temperature and pressure.

The full loadings of benzene and the interaction energy for most probable distributions (Table 2) indicate that these four models do not show noticeable differences. Moreover, four mass clouds for benzene molecules are similar, indicating that the minor distortion of the zeolite framework does not have 

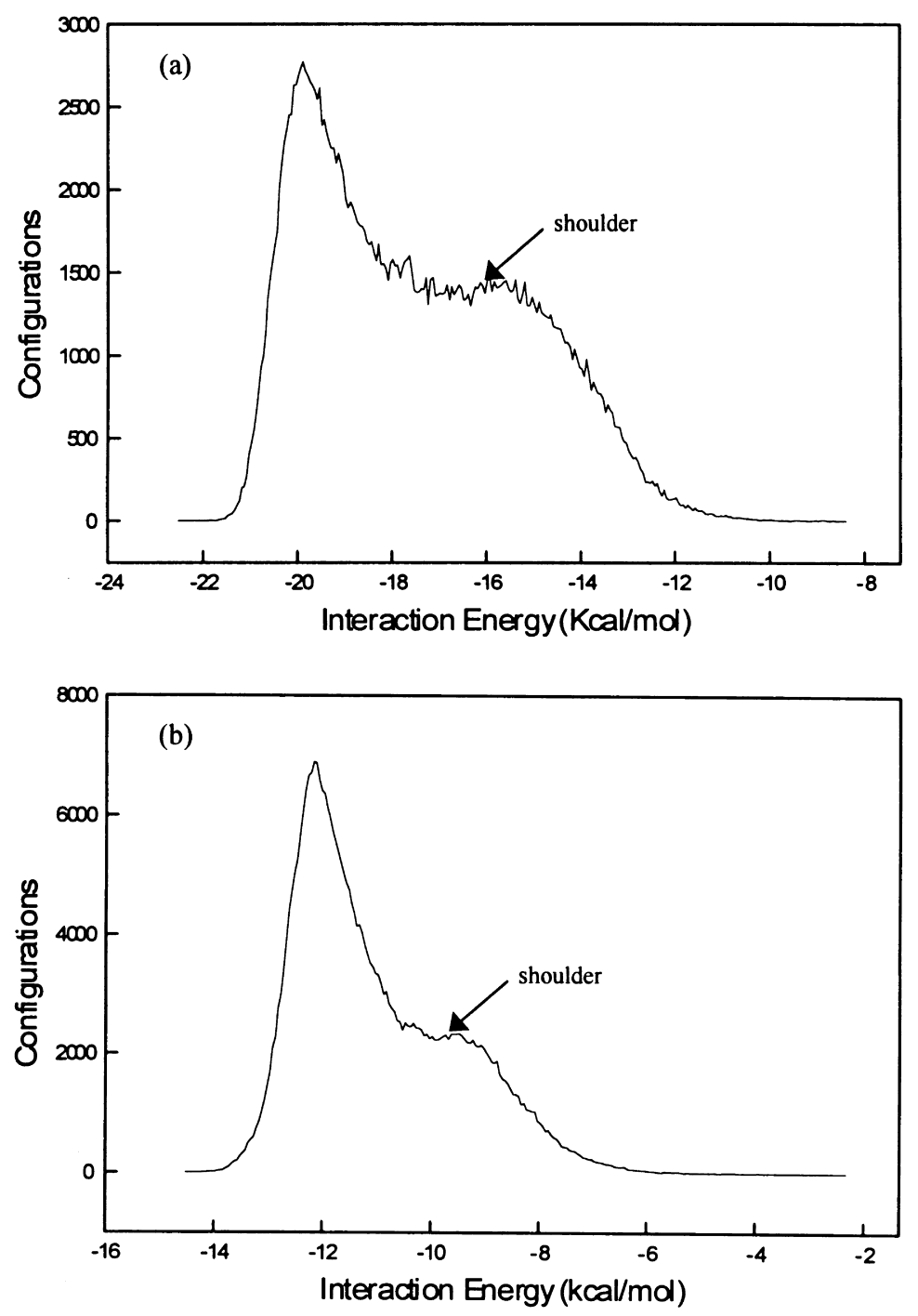

Fig. 3. (a) Benzene/zeolite potential energy distribution. (b) Propylene/benzene potential energy distribution.

considerable effect on the simulation results. So in this paper, the crystallographic structure of ITQ-1 is adopted and the zeolite flexibility is ignored.

\subsection{The diffusions of benzene in the two independent channel systems}

The evolution of the loading and energy of benzene shows that after $6 \times 10^{6}$ simulation steps, the equilibration has been achieved. At $300 \mathrm{~K}$ and $1 \mathrm{~atm}$, the full loading of benzene is about 63 molecules per 8
ITQ-1 unit cells. Fig. 3(a) depicts the energy distribution of interaction between benzene and ITQ-1 zeolite at full loadings. The energy distribution is roughly single-peaked, with a maximum around $-19.9 \mathrm{~kJ} \mathrm{~mol}^{-1}$, and a shoulder from $-17.5--15.0 \mathrm{~kJ} \mathrm{~mol}^{-1}$.

In order to characterize the location of the adsorbed molecules in the ITQ-1 zeolite, several mass clouds were depicted. As a powerful analysis tool, the mass cloud shows the preferred positions of the sorbates in the zeolite. The mass cloud of benzene, with respect to 


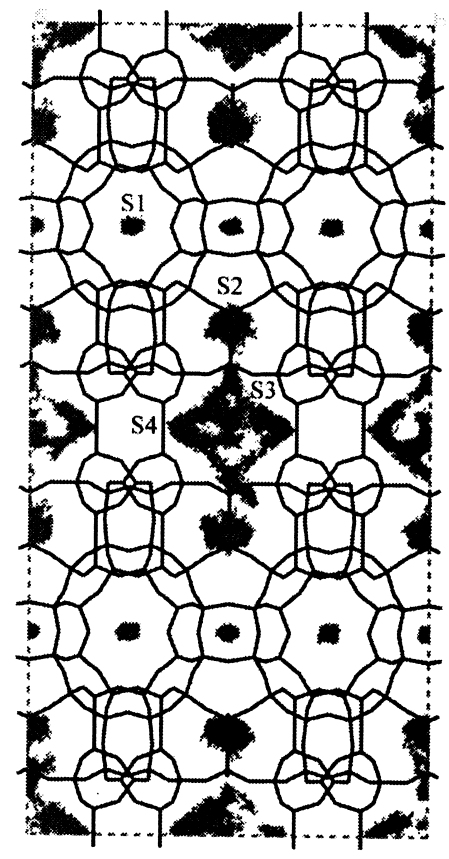

(a)

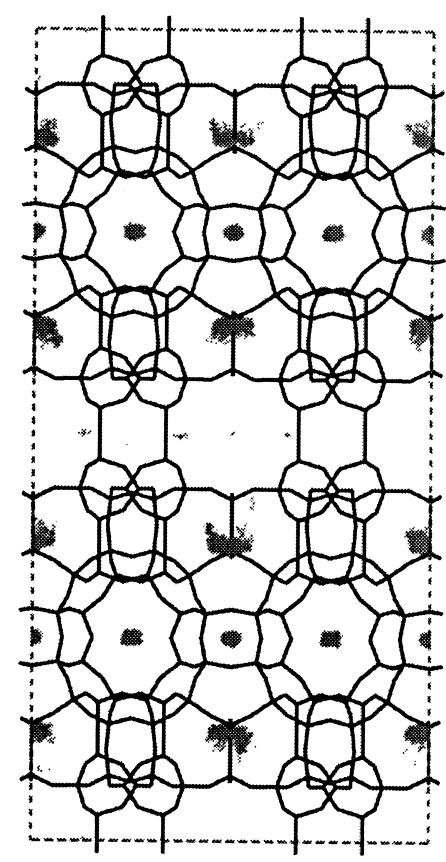

(b)

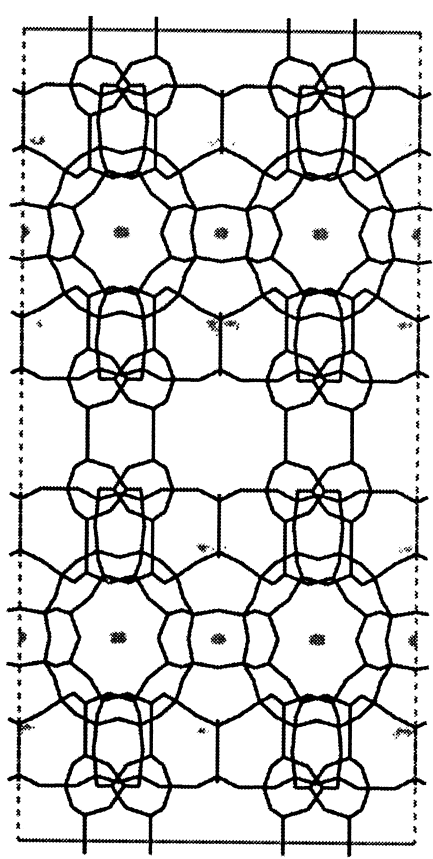

(c)

Fig. 4. (a) Mass cloud for benzene in the ITQ-1 cavities. (b) Mass cloud for benzene with interaction energy ranging from -100 to $-18 \mathrm{~kJ} \mathrm{~mol}^{-1}$. (c) Mass cloud for benzene with interaction energy ranging from -100 to $-20 \mathrm{~kJ} \mathrm{~mol}^{-1}$.

the zeolite famework, is shown in Fig. 4. The center of mass for each sorbate molecule in each configuration is displayed as a dot in the model space. From Fig. 4(a), it can be noted that the spatial distribution of benzene is roughly territorial, which can be divided into four regions: one in the 10-MR channels and three others in the 12-MR supercages, which have been named as S1, S2, S3 and S4. The S1 site is located in the 10-MR channels. The $\mathrm{S} 2$ site is observed near the 10-MR facing the 6-MR in supercage, while both the S3 and S4 sites are close to the central part of the 12-MR supercages. The distribution of benzene in the present simulations is somewhat similar to the trajectories of benzene derived from the previous molecule dynamics simulation performed by Sastre et al. [12].

Fig. 4(c) depicts the mass cloud of benzene with interaction energy ranging from -100 to $-20 \mathrm{~kJ} \mathrm{~mol}^{-1}$ (the benzene molecules with interaction energy lower than the most probable energy). It can be observed that the benzene molecules with relatively lower interaction energy are almost adsorbed close to the S1 site. Fig. 4(b) shows the mass cloud ranging from -100 to $-18 \mathrm{~kJ} \mathrm{~mol}^{-1}$; besides benzene adsorbed close to the $\mathrm{S} 1$ site, some benzene molecules near the S2 site also fall into this energy interval. Comparing three mass clouds in Fig. 4, it is obvious that the interaction energy of benzene at the S3 and S4 sites is mainly higher than $-18 \mathrm{~kJ} \mathrm{~mol}^{-1}$.

The interaction energy of benzene adsorbed close to the $\mathrm{S} 1$ site is only about $-20 \mathrm{~kJ} \mathrm{~mol}^{-1}$, but these molecules seem to move within a restricted area in the 10-MR channels. Although there is a high degree of tortuosity in the circular channels, the circular 10-MR channels in ITQ-1 are so small (only $4.0 \AA \times 5.5 \AA$ ) that it becomes difficult for benzene to diffuse through the 10 -MR sinusoidal channels of ITQ-1. Our results on the S1 site occupancy are in good agreement with the recent molecular dynamics simulations of the diffusion of benzene and propylene in ITQ-1 [12], which indicates that the benzene molecules move within a restricted area around the minimum energy position from the trajectories of molecular dynamics. 


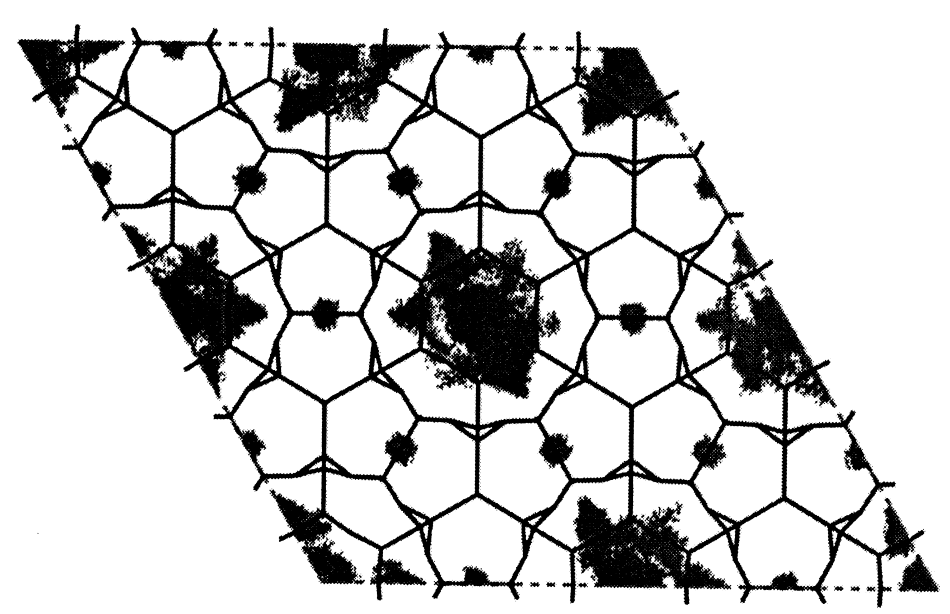

Fig. 5. Mass cloud for benzene in the ITQ-1 cavities along the $x y$ orientation.

For the product of the alkylation of benzene with propylene, it will be more difficult for the larger cumene to migrate through 10-MR channel systems. The previous results of Perego's study compared the diffusion of cumene in MCM-22 and ZSM-5, where the activation energies had been calculated to be 90.0 and $18.6 \mathrm{kcal} \mathrm{mol}^{-1}$ in the sinusoidal channels of the MWW structure and in the channels of ZSM-5, respectively. The combination of the present work and the previous simulations suggests that the benzene and the larger cumene molecules will find it very difficult to penetrate through the 10-MR sinusoidal channels.

At the S2 site, the benzene molecules also possess relatively lower interaction energy, although a little higher than those at the S1 site, which distribute in a generally localized manner. At the S2 site, the plane of benzene is observed to prefer being parallel with benzene near the S1 site. We assumed that the benzene molecules at the S2 and S3 sites would produce relatively strong aromatic stacking interactions.

The other two interesting sites are located near the center of the 12-MR supercages (the S3 and $\mathrm{S} 4$ sites). In our model, the interaction energy of benzene adsorbed close to those two sites is higher than $-18 \mathrm{kcal} \mathrm{mol}^{-1}$. It is apparent from Fig. 4(a) that the benzene molecules near the S3 and S4 sites are considerably delocalized in the vicinity of their preferred sites of adsorption, which are quite different from those near the S1 and $\mathrm{S} 2$ sites.

Each supercage is connected to six other supercages through 10-MR windows, and therefore, intercage motion is, in principle, possible. But partly due to the size and the position of the 10MR interconnecting windows, the benzene molecules near them should be energetically unfavorable, and relatively high activation energy must be needed for benzene molecules to migrate from one supercage to another through 10-MR windows. In Fig. 4(a), benzene is not observed in the 10-MR interconnecting region, so it can be concluded that the migration of benzene mainly happens in the same supercage, and the intercage motion is somewhat difficult from the viewpoint of interaction energy. Anyway, the migration of benzene along 12-MR cavities is much easier than that along 10-MR channels.

\subsection{The diffusions of propylene in the two independent channel systems}

The evolution of the loading and energy of benzene shows that propylene achieves the equilibrium faster than benzene. At $300 \mathrm{~K}$ and $1 \mathrm{~atm}$, the full loading of propylene is about 83.7 molecules per 8 ITQ-1 unit cells. The mean total interaction energy between the sorbates and the zeolite is nearly $-9.41 \times 10^{2} \mathrm{kcal} \mathrm{mol}^{-1}$. Fig. 3(b) depicts the energy distribution of interaction energy 


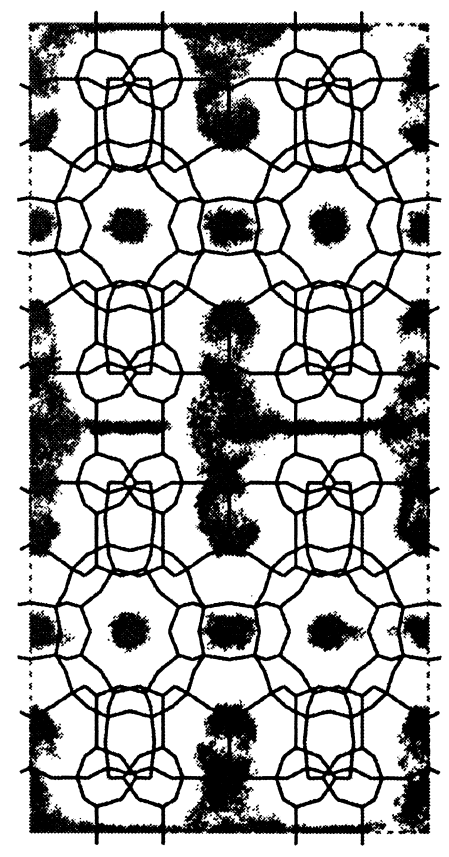

(a)

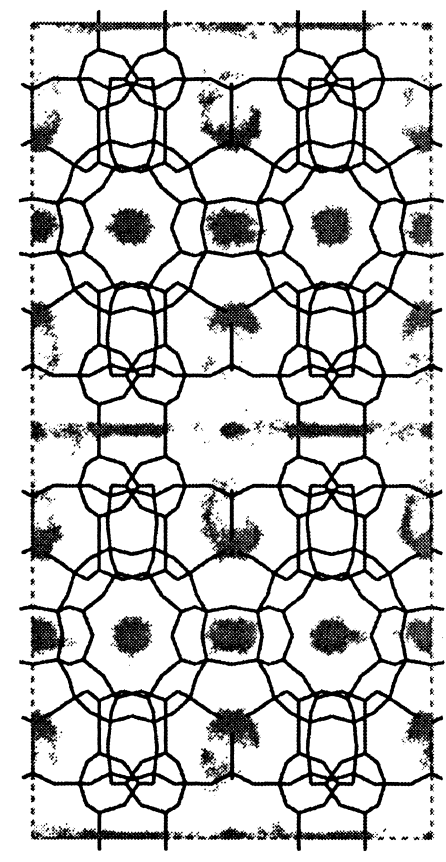

(b)

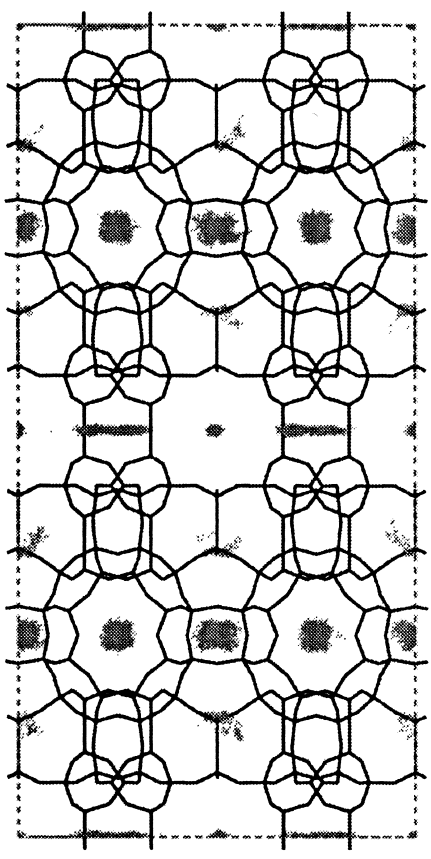

(c)

Fig. 6. (a) Mass cloud for propylene in the ITQ-1 cavities. (b) Mass cloud for benzene with interaction energy ranging from -100 to $-10.5 \mathrm{~kJ} \mathrm{~mol}^{-1}$. (c) Mass cloud for benzene with interaction energy ranging from -100 to $-12 \mathrm{~kJ} \mathrm{~mol}^{-1}$.

between propylene and ITQ-1 zeolite at full loading. The energy distribution of propylene is quite similar to that of benzene, roughly singlepeaked and an obvious shoulder. But the interaction energy for propylene is much higher than that for benzene, which means propylene may be more unfavorable in zeolite than benzene; moreover, the shoulder for propylene is not obvious.

The mass cloud of propylene indicates a completely different picture compared with that of benzene (Fig. 5). From Fig. 6, it can be seen that compared with benzene, the propylene molecules located in

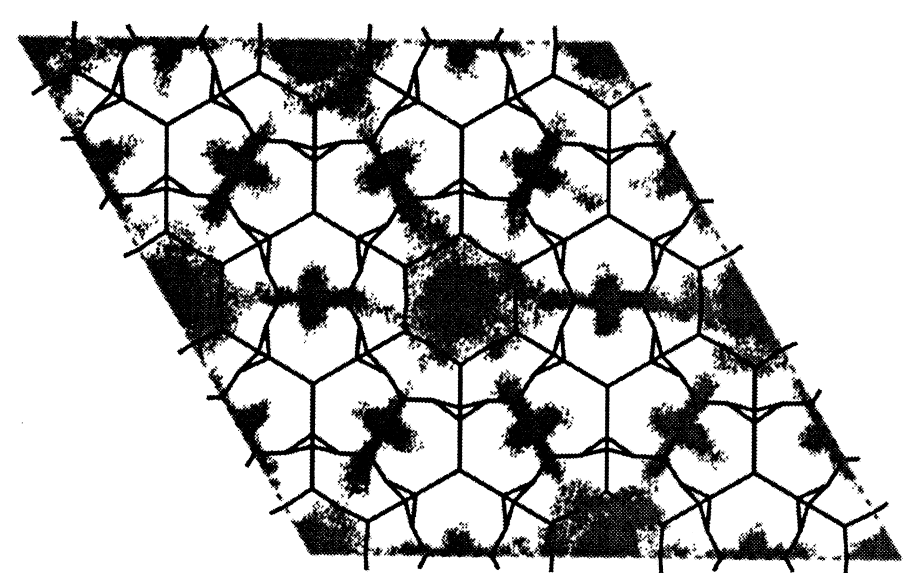

Fig. 7. Mass cloud for propylene in the ITQ-1 cavities along the $x y$ orientation. 


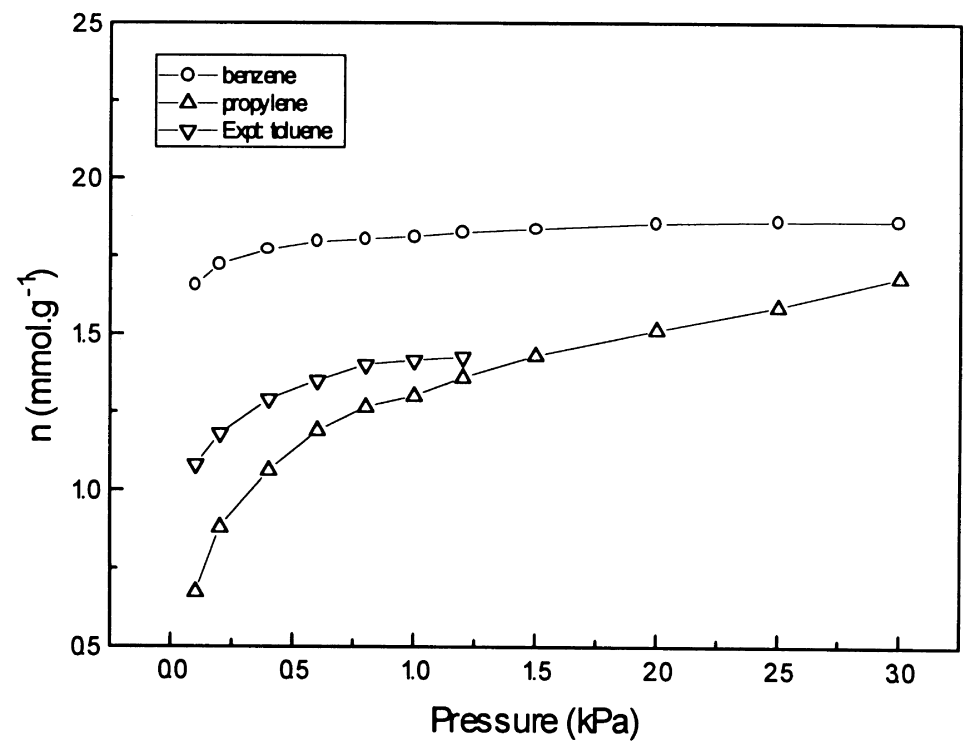

Fig. 8. Simulated adsorption isotherms of benzene and propylene at $315 \mathrm{~K}$ and experimental values for toluene.

the 10-MR channels are generally localized, but a much wider area is covered by the smaller propylene molecules. Careful inspection of Fig. 6 shows that the propylene molecules are not observed in the channel intersections, indicating that the channel intersections correspond to the locations of higher energy. Although propylene seems to move more freely in 10-MR channels compared to benzene, a certain activation energy seems to be needed for propylene to migrate through the 10-MR channels freely.

A completely different picture can be observed for propylene (Fig. 7) in the 12-MR supercages compared with benzene, where the propylene molecules almost fill all the possible positions in one supercage. It should be noted that the propylene molecules can be steadily located in the short 10-MR conducts around $3 \AA$ long, very different from the mass cloud of benzene. Fig. 6(b) shows the mass cloud of propylene with interaction energy ranging from -100 to $-12 \mathrm{~kJ} \mathrm{~mol}^{-1}$ (the sorbate molecules with interaction energy lower than the most probable energy), and it can be observed that the propylene molecules within that energetic interval are mainly distributed in the interconnecting area of 10-MR channels and the short 10-MR conducts. Obviously, the propylene molecules located in some areas between two adjacent 12-MR supercages are energetically favorable, which means that propylene can cross from one 12-MR supercage to another easily and does not even require any activation energy.

Considering the localization and adsorption of propylene and benzene in two separate channel systems, it can be concluded that the reaction will mainly occur in supercages, not in 10-MR channels, because the benzene molecules and the larger product of alkylation are very difficult to migrate through 10-MR channels. Moreover, it can be concluded that the alkylation on MCM-22 will be partly diffusion-controlled, and the diffusion of benzene in 12-MR supercages and especially the out-diffusion of cumene will be the controlling steps of the alkylation reaction.

\subsection{Predictions of adsorption isotherms for benzene and propylene}

In order to investigate the adsorption behavior of benzene and propylene thoroughly, a series of simulations has been performed to get the adsorption isotherms. In order to be compared with some experimental results, the temperature of the simulations is set at $315 \mathrm{~K}$, and the pressure ranges from 0.0 to $3.0 \mathrm{kPa}$. The calculated adsorption isotherms of pure propylene and benzene in ITQ-1 at $315 \mathrm{~K}$ are shown 


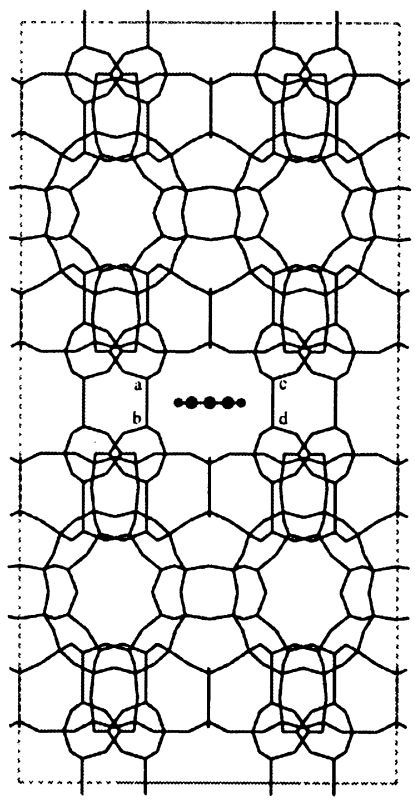

(a)

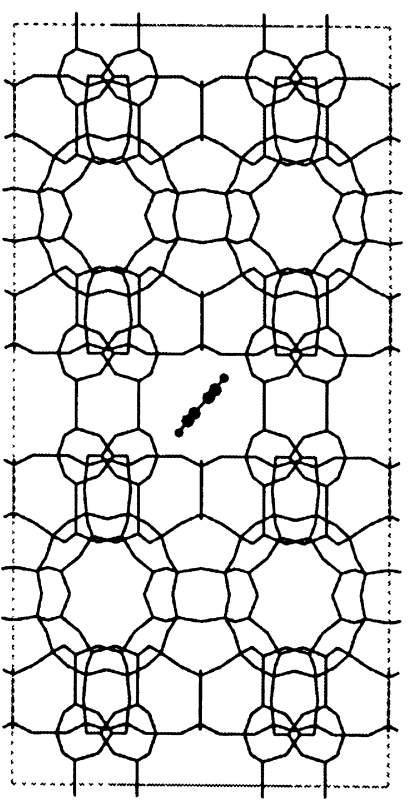

(b)

Fig. 9. Two energy minimum conformers of benzene near the abcd plane in the 10-MR window. The benzene molecule is represented with a ball-and-stick model.

in Fig. 8. Because the experimental isotherms for propylene and benzene are not available, the experimental isotherm of toluene is used for comparison [22]. Certainly, the isotherms of benzene and toluene cannot be quantitatively compared, but their structures are similar and their interactions with the zeolite lattice are only slightly different, so their adsorption behavior in the zeolite lattice should be similar to some extent. Only from the viewpoint of volume, benzene is relatively small, so its loadings should be remarkably higher than those of toluene, which can be well deduced from our simulations (Fig. 8). The experiments of infrared spectroscopy and adsorption-microcalorimetric studies have been applied to the adsorption-diffusion behavior of toluene, metaand ortho-xylene, and 1,2,4-trimethylbenzene with different kinetic diameters in MCM-22 [22], and the adsorption isotherms have validated that the zeolite uptake significantly relies on the size of the adsorbate molecules. It can be observed that the uptake of $m$ xylene is about half the value of toluene. The value of $o$-xylene is much lower and approximately the same as that of 1,2,4-trimethylbenzene. From our simula- tions, it can be noted that the uptake of benzene is much higher than the value of toluene, which also accords with previous research [22].

The most obvious difference between the adsorption isotherms of benzene and propylene is that the loadings of propylene are significantly larger than those of benzene at low pressure, which seems not to agree with the laws derived from the isotherms of some aromatic compounds. For aromatic compounds, their structures do not exhibit significant difference, and interactions between sorbate/zeolite and sorbate/ sorbate should be similar, so the adsorbed amounts are mainly concerned with the size of the sorbate molecules. In the case of benzene and propylene, their structures are quite different; besides the geometric factor, the energetic factor will also contribute a lot to their loadings. The comparison of benzene and propylene reveals that the interaction energy between benzene and the zeolite framework is obviously lower than that between propylene and the zeolite framework. In previous simulations at $350 \mathrm{~K}$ and $1 \mathrm{~atm}$, it has been validated that the interaction energy for propylene is much higher than that of benzene, 


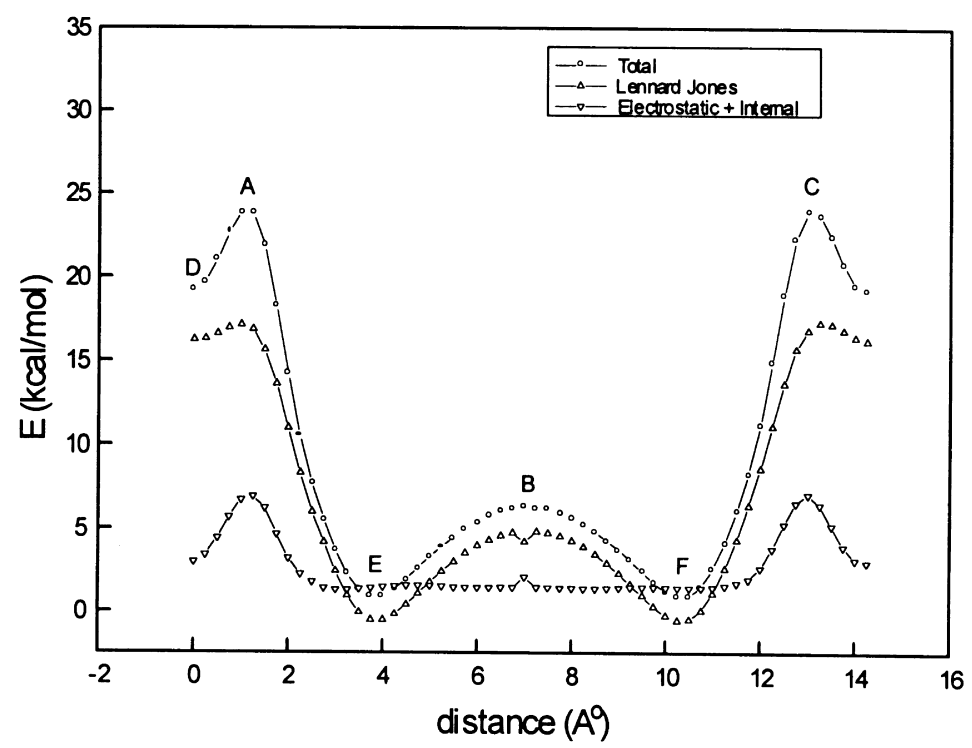

Fig. 10. Energy profile for benzene in ITQ-1 as a function of the distance between the center-of-mass of the molecule and the center of the $a b c d$ plane while it is pulled from cage to cage. Points A-C correspond to the potential maximum position, and points D-F correspond to the potential minimum position.

which means that in zeolite cavities propylene may be more unfavorable than benzene. So in some conditions, especially under low pressure, the unfavorable energy will make the uptake of propylene lower than that of benzene.

\subsection{Migration of benzene through the interconnecting 10-MR windows}

Previous calculations have hypothesized that the reaction will mainly happen in 12-MR supercages and the alkylation process will be partly affected by diffusion of reactants especially benzene and its products. Obviously, the intercage motion of benzene will be the most important factor to be considered. In order to get a clearer picture about the migration of benzene from one supercage to another, a single benzene molecule has been observed following a very simple path connecting two 12-MR supercages. During the calculations, the zeolite structure is rigid, but the conformational flexibility of benzene is allowed to be considered, so the potentials include three terms: Lennard-Jones and Coulomb potential between zeolite and benzene, plus the internal potential of benzene.

Energy minimizations under constraints were used for the systematic search for local minima. First, considering that during the migration process, the potential barrier may be near the 10-MR interconnecting areas, the benzene molecule was placed near the 10-MR window as the starting point of its path. Atoms a-d (Fig. 9) are four Si atoms in the 10-MR window, which construct a plane. First, the gravity centers of the plane and the benzene molecule were superimposed. Near the starting point, the benzene molecule was rotated systematically and the energy minimizations were performed to find the potential minima. The results indicate that near this point, two potential minima for benzene can be found (Fig. 10). Moreover, from the viewpoint of energy, the first conformer is preferred. Second, we forced the benzene molecule to translate through the supercage along the axis of symmetry, and a simple energy minimization strategy was applied to optimize the conformation of the benzene molecule to find the local potential minima. Third, we divided the corresponding path into small steps $0.25 \AA$ distant from each other, and for all the corresponding conformations, we calculated the interaction energy between the benzene molecules and the zeolite framework. Fig. 11 shows the minimum guest-host interaction energy as a function of the distance between the center-of-mass of benzene and 


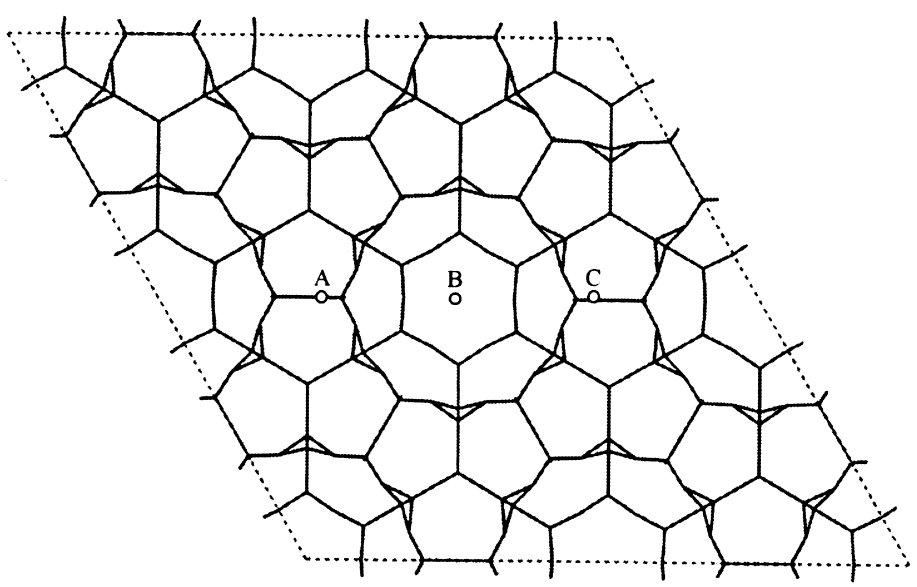

Fig. 11. Top view of the ITQ-1 structure showing the potential barriers of benzene if it is pulled from cage to cage. Points A-C correspond to the potential maximum position.

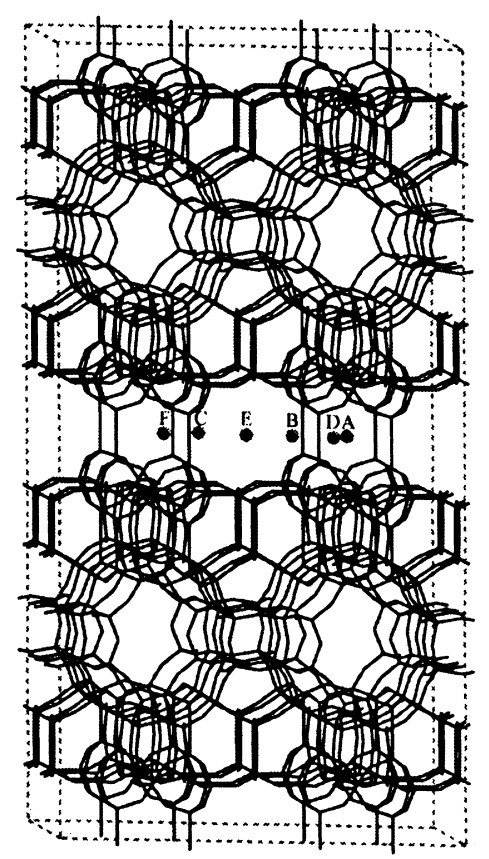

Fig. 12. Side view of the ITQ-1 structure showing the critical points $(\mathrm{A}-\mathrm{F})$ in the diffusion path followed by the benzene molecule as it is pulled from cage to cage. Points A-C correspond to the potential minimum position, and points $\mathrm{D}-\mathrm{F}$ correspond to the potential maximum position. that of the plane $a b c d$. Because of the symmetry of the zeolite, the energy function is mirror symmetric with respect to the plane $a b c d$. Only three crystallographically different types of energetic minima are observed.

The minima at $0 \AA$ corresponds to the starting point, while 3.75 and $10.25 \AA$ seem to correspond to the S4 adsorption site inside the supercages. Meanwhile, there exist three potential barriers along the pathway: A, B, and C (Fig. 11), among which the potentials at $\mathrm{A}$ and $\mathrm{C}$ are much higher than that at B. The energy profile of benzene as it is pulled from cage to cage in the ITQ-1 structure is similar to the previous simulations by Sastre et al. [12]. Obviously, the potential barriers should control the diffusion of benzene through 10-MR windows. From Fig. 11, it can be seen that $\mathrm{A}$ and $\mathrm{C}$ points are all near 10-MR windows, and they are symmetric to plane abcd. Moreover, the separate contributions to the activation energy have been calculated (Fig. 10). The first contribution comes from the Lennard-Jones energy, and the second one comes from the electrostatic interaction between the benzene molecule and the zeolite framework plus the conformational energy of benzene. As in Fig. 10, the Lennard-Jones potential obviously contributes more than the electrostatic potential (the internal conformational energy of benzene can be ignored). The energy necessary to cross from cage to cage is around $24 \mathrm{kcal} \mathrm{mol}^{-1}$. Previous calculation results from molecular dynamics [12] have predicted 
that the values are ranging from 15 to $20 \mathrm{kcal} \mathrm{mol}^{-1}$, which generally agrees with our predictions. The deviation mainly comes from the different potential parameters and different partial charges adopted in this paper.

Along the energy profile, there exists another potential barrier at position $\mathrm{B}$, which is just in the center of the 12-MR supercage. If we treat the lowest energy minimum in the benzene path as the adsorption S4 site, therefore, one benzene molecule that migrates from one $\mathrm{S} 4$ site to another in the same 12-MR supercage will only need $6 \mathrm{kcal} \mathrm{mol}^{-1}$, which is much lower than that for intercage migration. That is to say, the migration of benzene mainly happens in the same supercage and the intercage motions should need certain activation energy.

Certainly, the diffusion path of the benzene molecule is very simple and ideal (Fig. 12), and it only corresponds to very low loadings of benzene. In normal conditions, especially for high loadings, the presence of the interaction energy of the sorbates will influence the activation energy greatly, but the present study can also afford some useful information about the diffusion behavior. Because certain large activation energy is needed for benzene molecules to cross from one cage to another, increasing the temperature would possibly increase the probability of observing benzene intercage motions.

\section{Conclusions}

GCMC simulations have been performed to simulate the location and adsorption of benzene and propylene in a purely siliceous MWW structure (ITQ-1). Two separate simulation processes have been carried out to explore the locations and possible adsorption sites in each channel system of the ITQ- 1 at $300 \mathrm{~K}$ and $1 \mathrm{~atm}$. From calculation results of different distorted models, it can be concluded that the minor change of the zeolite framework does not introduce noticeable effects on the simulation results. The mass clouds from GCMC simulations indicate that benzene and propylene show high localization in 10-MR channels, which is due to the small size of the 10-MR openings of the sinusoidal systems. In 12-MR supercages, the benzene and propylene molecules show quite different mobilities. The spatial distribution of benzene in a
12-MR supercage can be clearly divided into three sites of adsorption: one site near the 10-MR facing the 6-MR in supercage and the other two near the center of the 12-MR supercages. So we can conclude that the migration of benzene molecules mainly happens in the same supercage, and the intercage motions should need certain activation energy. In the case of propylene, the sorbate molecules not only almost fill all the possible positions in one supercage, but also can be steadily located in the short 10-MR conducts around $3 \AA$ long, which means that propylene can cross from one supercage to another very easily and does not require obvious activation energy. The adsorption isotherms of benzene and propylene at $315 \mathrm{~K}$ and $0-3.0 \mathrm{kPa}$ have been predicted, and the adsorption isotherm of benzene coincides with the trend from experiments on a series of aromatic compounds. At low pressure, the loadings of propylene are significantly lower than those of benzene, which are mainly caused by the relatively unfavorable interaction energy between propylene and the zeolite framework.

From the calculations for the activation energy of a benzene molecule in the intercage process through 10MR windows, it can be found that at the simulated conditions the thermal energy allows the mobility of benzene inside the cavity but not from one 12-MR cavity to another. By analyzing the locations of benzene and propylene in the ITQ-1 framework, the alkylation of benzene and propylene will mainly happen in 12-MR supercages at the external surface or close to the external surface.

\section{Acknowledgements}

This project is supported by NCSF $29992590-2$ and 29573095.

\section{References}

[1] P.R. Pujado, J.R. Salazar, C.V. Berger, Hydrocarbon Process. 55 (1976) 91.

[2] H. Miki, US Patent, 4347393, 1982.

[3] E.K. Jones, D.D. Dettner, US Patent, 2860, 1958.

[4] A. Wood, Chem. Week 17 (1994) 34.

[5] W.W. Kaeding, R.E. Holland, J. Catal. 19 (1988) 212. 
[6] J. Chen, Wordwide Solid Acid Process Conference, Houston, TX, November 14, 1993.

[7] K.H. Chandavar, S.G. Hedge, S.B. Kulkarni, P. Ratnasamy, J. Chem. Technol. Biotechnol. 34A (1984) 165.

[8] J.C. Cheng, T.F. Degnan, J.S. Beck, Y.Y. Huang, M. Kalyanaraman, J.A. Kowalski, C.A. Loehr, D.N. Mazzone, Stud. Surf. Sci. Catal. 121 (1999) 53.

[9] M.E. Leonowicz, J.A. Lawton, S.L. Lawton, M.K. Rubin, Science 24 (1994) 1910.

[10] M.K. Rubin, P. Chu, US Patent, 4954325, 1990.

[11] C. Perego, S. Amarilli, R. Millini, G. Bellusi, G. Girotti, G. Terzoni, Microporous Mater. 6 (1996) 395.

[12] G. Sastre, C.R.A. Catlow, A. Corma, J. Phys. Chem. 103 (1999) 5187.

[13] M.A. Camblor, A. Corma, M.J. Diaz-Cabanas, C. Baerlocher, J. Phys. Chem. 102 (1998) 44.

[14] E.D.V. Burchart, PhD thesis, 1992.
[15] S.L. Mayo, B.D. Olafson, W.A. Goddard, J. Phys. Chem. 94 (1990) 8897.

[16] Cerius ${ }^{2}$ Simulation Tools User Reference, Molecular Simulation Inc., San Diego, CA, 1998.

[17] Mopac 7.0 User Guide, Quantum Chemistry Program Exchange (QCPE), Indiana University, USA, 1993.

[18] Q.S. Randall, T.B. Alexis, N.T. Doros, J. Phys. Chem. 97 (1993) 13742.

[19] P.A. Van Tassel, H.T. Davis, A.V. Mccormick, J. Chem. Phys. 98 (1993) 4173.

[20] M.A. Camblor, A. Corma, C. Baerlocher, J. Phys. Chem. 102 (1998) 44.

[21] M. Czjzek, H. Fuess, T. Vogt, J. Phys. Chem. 95 (1991) 5255.

[22] A. Coma, A. Corell, J. Pérez-Pariente, J.M. Guil, R. GuilLópez, S. Nicolopoulus, J. Gonzalez-Calbet, M. Vallet-Regi, Zeolite 17 (1996) 7. 